\title{
Comparison of National Comprehensive Cancer Network and European Position Statement protocols for nodule management in low-dose computed tomography lung cancer screening in a general Chinese population
}

\author{
Yihui Du ${ }^{1 \#}$, Yanju Li ${ }^{2 \#}$, Monique D. Dorrius ${ }^{1,3}$, Grigory Sidorenkov ${ }^{1}$, Marleen Vonder ${ }^{1}$, \\ Rozemarijn Vliegenthart ${ }^{3}$, Marjolein A. Heuvelmans ${ }^{1}$, Xiaonan Cui ${ }^{2}$, Zhaoxiang Ye $^{2}$, \\ Geertruida H. de Bock ${ }^{1}$
}

${ }^{1}$ Department of Epidemiology, University Medical Center Groningen, University of Groningen, Groningen, The Netherlands; ${ }^{2}$ Department of Radiology, Tianjin Medical University Cancer Institute and Hospital, National Clinical Research Centre for Cancer, Key Laboratory of Cancer Prevention and Therapy, Tianjin's Clinical Research Center for Cancer, Tianjin, China; ${ }^{3}$ Department of Radiology, University Medical Center Groningen, University of Groningen, Groningen, The Netherlands

Contributions: (I) Conception and design: Z Ye, GH de Bock, R Vliegenthart, MA Heuvelmans; (II) Administrative support: Z Ye, GH de Bock, G Sidorenkov, M Vonder; (III) Provision of study materials or patients: Z Ye, Y Li, X Cui; (IV) Collection and assembly of data: Y Li, MD Dorrius, X Cui; (V) Data analysis and interpretation: Y Du, Y Li, MD Dorrius, G Sidorenkov, M Vonder; (VI) Manuscript writing: All authors; (VII) Final approval of manuscript: All authors.

"These authors contributed equally to this work.

Correspondence to: Zhaoxiang Ye. Department of Radiology, Tianjin Medical University Cancer Institute and Hospital, National Clinical Research Centre for Cancer, Key Laboratory of Cancer Prevention and Therapy, Tianjin's Clinical Research Center for Cancer, Tianjin 300060, China. Email: yezhaoxiang@163.com.

Background: Low-dose computed tomography (LDCT) lung cancer screening often refers individuals to unnecessary examinations. This study aims to compare the European Position Statement (EUPS) and National Comprehensive Cancer Network (NCCN) protocols in management of participants at baseline screening round.

Methods: LDCT lung cancer screening was prospectively performed in a Chinese asymptomatic population aged 40-74 years. A total of 1,000 consecutive baseline LDCT scans were read twice independently. All screen-detected lung nodules by the first reader were included. The first reader manually measured the diameter of lung nodules (NCCN protocol), and the second reader semi-automatically measured the volume and diameter (EUPS volume and diameter protocols). The protocols were used to classify the participants into three management groups: next screening round, short-term repeat LDCT scan and referral to a pulmonologist. Groups were compared using Wilcoxon test for paired samples. Number of lung cancers by protocols was provided.

Results: Of the 1,000 participants (61.4 46.7 years old), 168 lung nodules in 124 participants were visually detected and manually measured in the first reading, and re-measured semi-automatically. Applying the NCCN protocol, EUPS volume and diameter protocol, the proportion of referrals among all participants was $0.6 \%, 1.9 \%$, and $1.4 \%$, respectively. The proportion of short-term repeat scans was $4.5 \%, 9.7 \%$ and $4.5 \%$, respectively. Among the 10 lung cancer patients, one would have been diagnosed earlier if the EUPS volume protocol would have been followed.

Conclusions: In a first round screening in a Chinese general population, the lower threshold for referral in the EUPS protocol as compared to the NCCN protocol, leads to more referrals to a pulmonologist, with the potential of earlier cancer diagnosis. The EUPS volume protocol recommends fewer participants to shortterm repeat LDCT scan than the EUPS diameter protocol. Follow-up studies should show the impact of both protocols on (interval) cancer diagnosis. 
Keywords: Lung nodule; mass screening; diameter; volume; low-dose computed tomography (LDCT)

Submitted Aug 09, 2021. Accepted for publication Oct 14, 2021.

doi: $10.21037 /$ jtd-21-1312

View this article at: https://dx.doi.org/10.21037/jtd-21-1312

\section{Introduction}

Screening for lung cancer by low-dose computed tomography (LDCT) has been shown to prevent premature death by detecting developing cancers at an early stage (1). The National Lung Screening Trial (NLST) and the Dutch-Belgian Randomized Lung Cancer Screening Trial (NELSON) demonstrated a reduction in lung cancer mortality and showed the benefit of LDCT screening for lung cancer in high-risk populations (2,3).

A main challenge in LDCT lung cancer screening is that the majority of screen-detected lung nodules are benign, which results in a large number of unnecessary short-term LDCT repeat scans and referrals to a specialist for further work up (4). The NLST study reported that $96 \%$ of the screen-detected nodules referred for work-up turned out to be benign, although any non-calcified nodules $\geq 4 \mathrm{~mm}$ were regarded as positive and suspicious for lung cancer in that study (2). The number of unnecessary scans and examinations depends on the definition of indeterminate and positive findings from the LDCT scan. There are several guidelines or recommendations for management of screen-detected lung nodules, such as the Lung CT Screening Reporting and Data System (Lung-RADS) developed by the American College of Radiology (5), and the guideline developed by the National Comprehensive Cancer Network (NCCN) (6), in which diameter thresholds are provided. In addition, based on the findings from NELSON study, the European Position Statement (EUPS) on lung cancer screening proposed volume thresholds for nodule management, in which the diameter thresholds companied (7). These guidelines were mainly developed for lung cancer screening in a high risk population, and proposed inconsistent definitions for intermediate and positive nodules which require short-term repeat LDCT scan and referral to a pulmonologist, respectively, thus it is necessary to evaluate how these guidelines differ in nodule management in a general population.

The ongoing Netherlands and China Big 3 diseases (NELCIN-B3) project was initiated with one of the objectives being evaluation of the performance of NELSON and NCCN protocols in terms of false positive rate in Chinese lung cancer screening setting (8). NELCIN-B3 study provided an opportunity for the intended comparison. The aim of the current study was to compare the rate of immediate referral and short-term repeat LDCT scan between NCCN and EUPS protocols in a Chinese screening setting.

We present the following article in accordance with the STARD reporting checklist (available at https://dx.doi. org/10.21037/jtd-21-1312).

\section{Methods}

In 2017, the NELCIN-B3 project was initiated aiming to improve early detection of lung cancer, chronic obstructive pulmonary disease and cardiovascular disease. Detailed study protocols have been published previously $(8,9)$. This prospective study was approved by the ethics committee of the leading institute of NELCIN-B3 study, namely the Committee on Ethics of Biomedicine Research of Second Military Medical University (8). Written informed consent was obtained from each participant. The NELCIN-B3 study has been registered on ClinicalTrials.gov with the registration number NCT03988322 and NCT03992833. This study conformed to the provisions of the Declaration of Helsinki (as revised in 2013).

\section{Study population}

In total 5,524 participants were prospectively recruited for LDCT lung cancer screening in the Tianjin Medical University Cancer Institute and Hospital (TJMUCIH) from June 2017 until July 2018. The inclusion criteria of participants have been described previously (8). Briefly, participants aged 40-74 years, residing in the Hexi district of Tianjin city for at least 3 years and without selfreported history of a malignant tumor were recruited. In the here presented analysis, the first LDCT scan of 

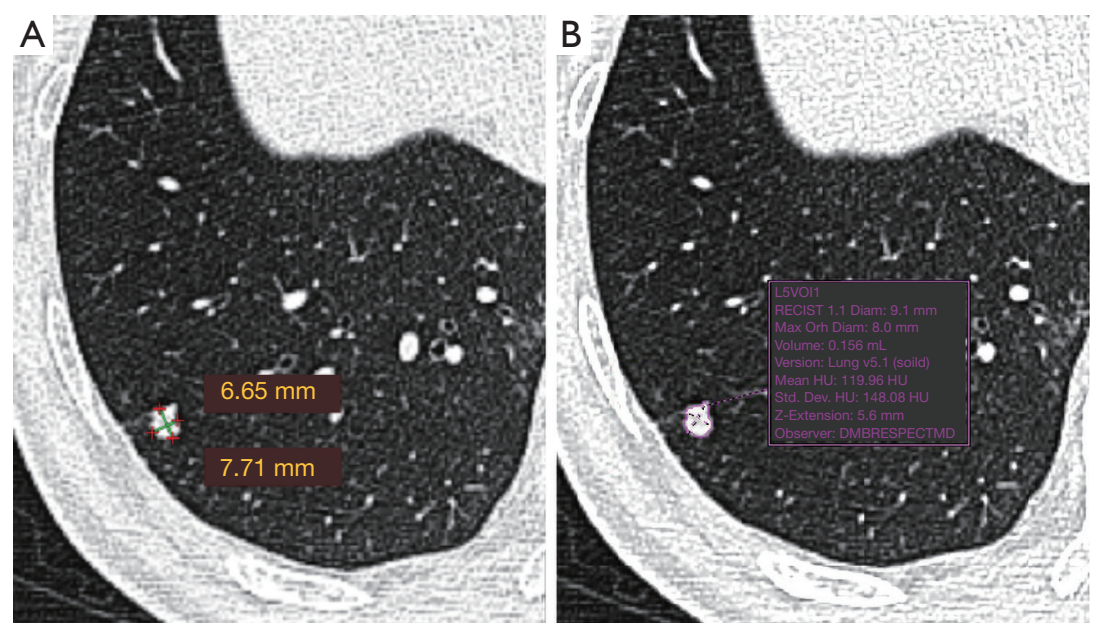

Figure 1 CT image with manual diameter measurement (A) and semi-automated volume and diameter measurement (B). CT, computed tomography.

1,000 consecutive participants at baseline were included.

\section{CT scan protocol}

The scan protocol has been described in detail previously (8). All scans were performed using the same CT system: Definition AS (Somatom Definition AS 64, Siemens Healthineers, Erlangen, Germany). Positioning of participants was head-first, supine, and with arms above the head. The CT scan was performed at end-inspiration in spiral scan mode, tube voltage of $120 \mathrm{kVp}$ and a reference tube current of $35 \mathrm{mAs}$. Data were reconstructed with medium-soft (D45F) and hard (B80F) kernels at a slice thickness and increment of $1.0 / 0.7 \mathrm{~mm}$, and with soft kernel (B30) at 2.0/1.0 $\mathrm{mm}$.

\section{Image reading and measurements}

The LDCT images were read first by Chinese radiologists and the visually-detected lung nodules were measured. The detected nodules were measured for a second time by a Dutch radiologist. The first reading was performed by one of the four Chinese resident radiologists (Juxiang Ma, Debei Ma, Zhijun Li and Qingqing Diao) who were specially trained for reading LDCT images for lung cancer screening, and supervised by one of two senior Chinese radiologists (YL and XC) at TJMUCIH. Images were interpreted both at lung and mediastinal window settings. A nodule was registered and measured if the diameter (mean of maximum and perpendicular diameter) $\geq 4 \mathrm{~mm}$. A nodule was considered part-solid when a nonsolid component was visible as well as a solid part in lung window setting (window-width: 1,200 HU, window-level: $-500 \mathrm{HU}$ ) and the solid part was also visible in mediastinal window setting (window-width: $320 \mathrm{HU}$, window-level: $35 \mathrm{HU})$. Measurement was performed in lung window setting. The maximum diameter of a nodule and its perpendicular diameter were measured manually on the axial plane in Carestream Picture Archiving and Communication Systems (PACS) v.11.0 (see the example in Figure 1). The second reading was performed by a radiologist (MDD) in The Netherlands with 10 years of experience in chest imaging, including experience in lung cancer screening at the University Medical Center Groningen, The Netherlands. The measurement of nodules was performed in lung window setting (window-width: 1,200 HU, windowlevel: $-500 \mathrm{HU}$ ) in the second reading. The second reader used semi-automated volumetry software (MM Oncology, Syngo.via VB30, Siemens), providing the volume as well as the diameter of lung nodules (see the example in Figure 1). The diameter was registered in three dimensions, including maximum axial diameter, perpendicular diameter and z-axis diameter.

\section{Nodule classification and participant management}

The NCCN (6) and EUPS (7) protocols for nodule management were used to classify the screen-detected lung nodules at baseline. The NCCN protocol was applied with the manual diameter measurements. The diameter used for 


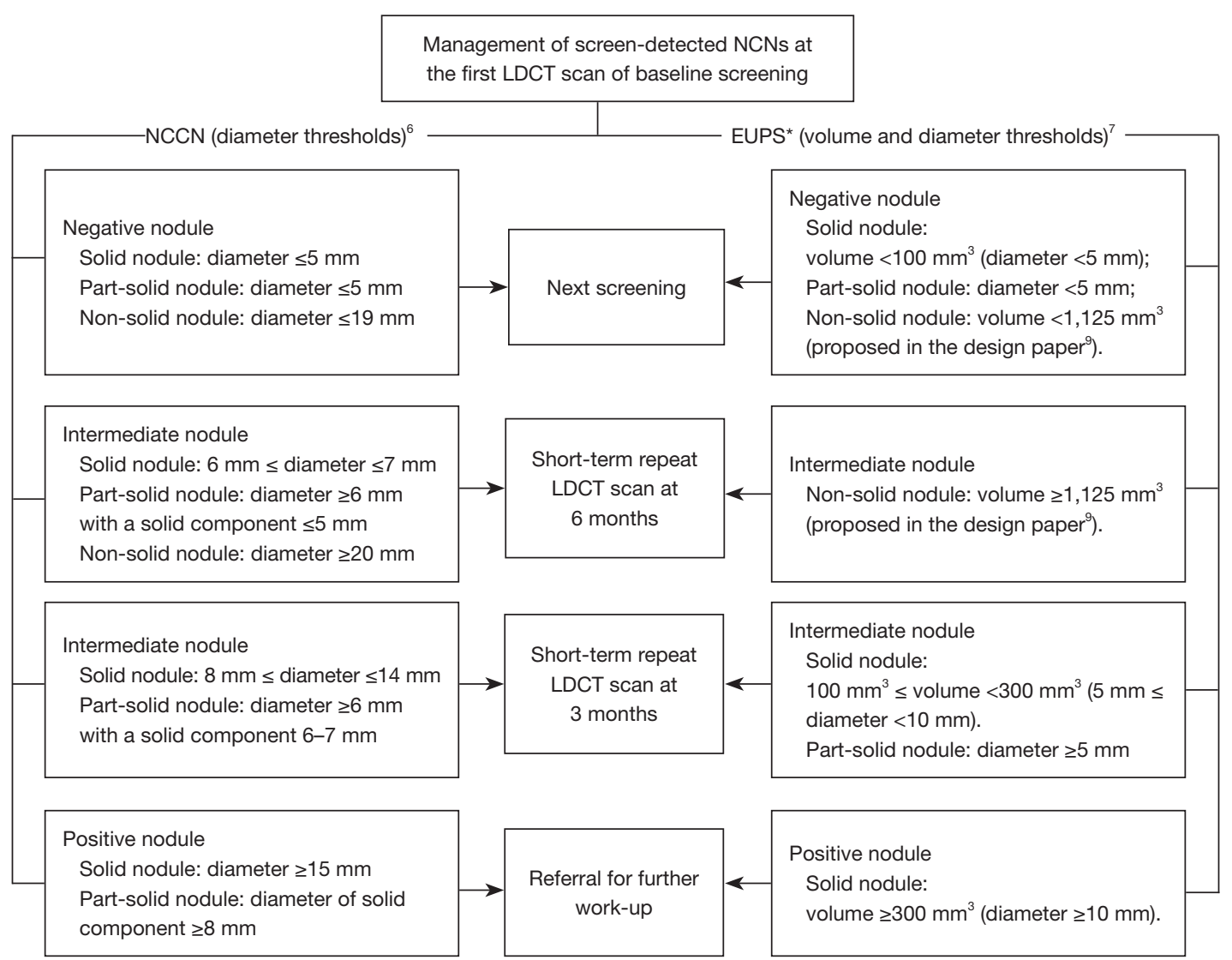

Figure 2 Management of screen-detected NCNs at initial LDCT scan in NCCN and EUPS protocols $(6,7,9) .{ }^{*}$, EUPS proposed the volume thresholds for solid nodule only. In this study, the volume thresholds were also applied for part-solid nodules. NCNs, noncalcified nodules; LDCT, low-dose computed tomography; NCCN, National Comprehensive Cancer Network; EUPS, European Position Statement.

management was the mean of the maximum diameter of the nodule and its perpendicular diameter measured at the axial plane. The mean diameter was rounded to the nearest whole number according to the NCCN protocol. The EUPS protocol provided both volume and diameter thresholds. The EUPS volume protocol was applied based on the semiautomatic volume measurements and the EUPS diameter protocol was applied based on the semi-automatic diameter measurement (mean diameter of maximum axial diameter and perpendicular diameter). In the local setting, the actual management of the screenees was based on the manually measured diameter (measurements on lung window setting except the solid component of part-solid was measured on mediastinal window setting) and NCCN recommendations.

The diameter thresholds proposed by the NCCN Lung Cancer Screening (version 2. 2018) in the Clinical Practice Guidelines in Oncology are presented in Figure 2. The volume and diameter thresholds of the EUPS protocol for the first scan at the baseline screening round were described in the EUPS paper (7). Briefly, for a solid nodule, a negative nodule (volume $<100 \mathrm{~mm}^{3}$, diameter $<5 \mathrm{~mm}$ ) leads to next screening round at 1 -year after the baseline scan, an intermediate nodule $\left(100 \mathrm{~mm}^{3} \leq\right.$ volume $<300 \mathrm{~mm}^{3}$, $5 \mathrm{~mm} \leq$ diameter $<10 \mathrm{~mm}$ ) results in recommendation for short-term repeat LDCT scan at 3 months after the baseline scan, and a positive nodule (volume $\geq 300 \mathrm{~mm}^{3}$, diameter $\geq 10 \mathrm{~mm}$ ) leads to referral to a pulmonologist for further work-up. EUPS paper only provided the volume thresholds for managing participants with solid nodule but not for part-solid and non-solid nodules. As described and rationalized in the design paper of NELCIN-B3 study (8), the same volume thresholds as solid nodules were applied for part-solid nodules; for a non-solid nodule with volume that exceeded $1,125 \mathrm{~mm}^{3}$, short-term repeat LDCT scan at 
6 months after baseline was proposed, and for a non-solid nodule with volume $<1,125 \mathrm{~mm}^{3}$, next screening round after 1-year was proposed (Figure 2).

Based on the size classification of screen-detected nodules, the management of participants at baseline included next screening, short-term repeat LDCT scan (including 6- and 3-month scan after the initial scan) and referral to a pulmonologist for further work-up. For participants with multiple nodules, the nodule with the largest diameter was applied to manage the individual. Alternatives for further work-up in the actual management of screen-detected suspicious nodules included positron emission tomography (PET) or CT scans, core needle biopsy, and primary resection.

\section{Collected features of lung nodules}

Consistency (solid, part-solid or non-solid), manual maximum diameter and perpendicular diameter of lung nodules were collected from the first reading, and semi-automated volume and semi-automated diameter measurement of the nodules were collected from the second reading. For part-solid nodules, the diameter and volume of the solid component were additionally collected. The diagnosis of lung cancer among all participants with baseline nodules by the time of the second round screening (including the second round screening results) was collected through the Hospital Information System of TJMUCIH or through contact by calling. The non-cancer status was confirmed based on the second round screening results.

\section{Sensitivity analysis}

In the NCCN guideline, the average diameter of lung nodules was rounded to the nearest whole number for nodule management. To assess the effect of applying the diameter measurement with one decimal fraction (not rounding to the nearest whole number) on nodule classification and participants management, a sensitivity analysis was performed.

\section{Statistical analysis}

With an estimated effect size of 0.19 in this study, a sample size of 1,000 and an alpha error of $5 \%$, the study will have $93 \%$ of power to detect the effect of the EUPS volume protocol on the classification to the group of short-term repeat CT or referral compared to the NCCN protocol.
The description of nodule size is presented in mean and standard deviation (SD), median and interquartile range (IQR), minimum and maximum. The classification of nodules and management of participants according to the NCCN and EUPS protocols is presented in frequency and percentage. The difference in participants classification between the two protocols was tested with Wilcoxon test for paired sample and $\mathrm{P}<0.05$ was considered statistically significant. All analyses were conducted in R Statistical Software (version 3.5.1).

\section{Results}

\section{Characteristics of participants and nodules}

Among the 1,000 consecutive participants at baseline, $55.0 \%(\mathrm{n}=550)$ were women. Mean age of the participants at baseline was $61.4 \pm 6.7$ years (men: $63.1 \pm 6.1$ years, women: $60.0 \pm 6.8$ years). There were $219(48.7 \%)$ current smokers, $95(21.1 \%)$ former smokers, $136(30.2 \%)$ never smokers in men, and $16(2.9 \%)$ current smokers, $3(0.5 \%)$ former smokers, $529(96.2 \%)$ never smokers in women.

In total, 168 non-calcified lung nodules of 124 participants were registered and measured. Of the 124 participants, $63(50.8 \%)$ were women. The mean age was $62.5 \pm 6.1$ years (men: $63.8 \pm 5.6$ years, women: $61.2 \pm 6.4$ years). Majority (85.7\%) of the screen-detected nodules were solid. For solid nodules, the median of the manual diameter measurement, semi-automated diameter measurement and semi-automated volume measurement was 5.0 (IQR: 4.4-6.0) mm, 6.2 (IQR: 5.3-7.8) $\mathrm{mm}$ and 92.5 (IQR: $61.0-162.2) \mathrm{mm}^{3}$, respectively (Table 1). The nodule classification by NCCN and EUPS protocols are presented in Table S1.

\section{Lung cancer diagnosis}

Among the 1,000 participants, by the time of the second round screening (including the second round screening results), 9 were diagnosed with lung cancer based on pathology results, 1 was diagnosed with suspected lung cancer by the radiologist but without confirmation from histopathology, 793 (79.3\%) were not diagnosed with lung cancer and 197 (19.7\%) were lost to follow-up. The 10 cancer patients included 8 diagnosed at the first screening round and 2 at the second screening round (Figure 3). The characteristics of the participants with lung cancer are presented in Table 2. 
Table 1 Description of the size of the screen-detected lung nodules at the initial LDCT scan for lung cancer screening

\begin{tabular}{|c|c|c|c|}
\hline Indicator & $\begin{array}{c}\text { Manual diameter } \\
\text { measurement* }(\mathrm{mm})\end{array}$ & $\begin{array}{l}\text { Semi-automated diameter } \\
\text { measurement }{ }^{\#}(\mathrm{~mm})\end{array}$ & $\begin{array}{l}\text { Semi-automated volume } \\
\text { measurement }\left(\mathrm{mm}^{3}\right)\end{array}$ \\
\hline \multicolumn{4}{|l|}{ Solid nodule $(n=144)$} \\
\hline Mean \pm SD & $5.0 \pm 4.7$ & $7.4 \pm 5.2$ & $600.7 \pm 3,528.1$ \\
\hline \multicolumn{4}{|c|}{ Part-solid nodule $(\mathrm{n}=5)$} \\
\hline \multicolumn{4}{|l|}{ Whole nodule } \\
\hline Median (IQR) & $10.6(8.5-14.9)$ & $11.8(11.8-17.6)$ & $991.0(606.0-1,082.0)$ \\
\hline Solid component & & NA & \\
\hline Median (IQR) & $7.0(6.0-11.0)$ & & $583.0(346.0-702.0)$ \\
\hline Mean \pm SD & $8.2 \pm 3.7$ & & $506.8 \pm 269.4$ \\
\hline Min & 4.0 & & 124.0 \\
\hline Max & 13.0 & & 702.0 \\
\hline \multicolumn{4}{|c|}{ Non-solid nodule $(n=19)$} \\
\hline Median (IQR) & $6.5(5.5-7.5)$ & $7.9(6.2-9.9)$ & $110.0(91.5-211.0)$ \\
\hline
\end{tabular}

*, the diameter was the mean of the maximum diameter of the nodule and its perpendicular diameter measured at axial plane; ", the diameter was the mean of the maximum axial diameter and orthogonal diameter of the nodule. LDCT, low-dose computed tomography; IQR, interquartile range; SD, standard deviation; NA, not available.

\section{Comparison of NCCN and EUPS protocols at participant level}

The proportion of referrals to a pulmonologist in the participants according to NCCN protocol was lower than that according to EUPS volume protocol ( $0.6 \%$ vs. $1.9 \%)$. The proportion of short-term repeat scans was similar according to the two protocols ( $4.5 \%$ vs. $4.5 \%$ ) (Table 3).

The proportion of referrals to a pulmonologist in the participants according to EUPS volume protocol and EUPS diameter protocol was similar (1.4\% vs. $1.9 \%)$. But the proportion of short-term repeat scans according to EUPS volume protocol was lower than the EUPS diameter protocol $(4.5 \%$ vs. $9.7 \%)$. The conclusion remained when restricted to participants with solid nodule (Table 3).

Among the 949 participants referred to the next screening round in the NCCN protocol, $6.3 \%$ (60 participants) and $1.7 \%$ (16 participants) would require a short-term repeat CT scan if applying the EUPS diameter and volume protocol, respectively. Among the 45 participants needing shortterm repeat CT according to the NCCN protocol, $22.2 \%$ (10 participants) and $28.9 \%$ (13 participants) would be referred to a pulmonologist if applying the EUPS diameter and volume protocol, respectively. The management differed significantly between the NCCN and EUPS protocols $(\mathrm{P}<0.001)$ (Table 4).

Regardless of which protocol was applied, 10 participants 


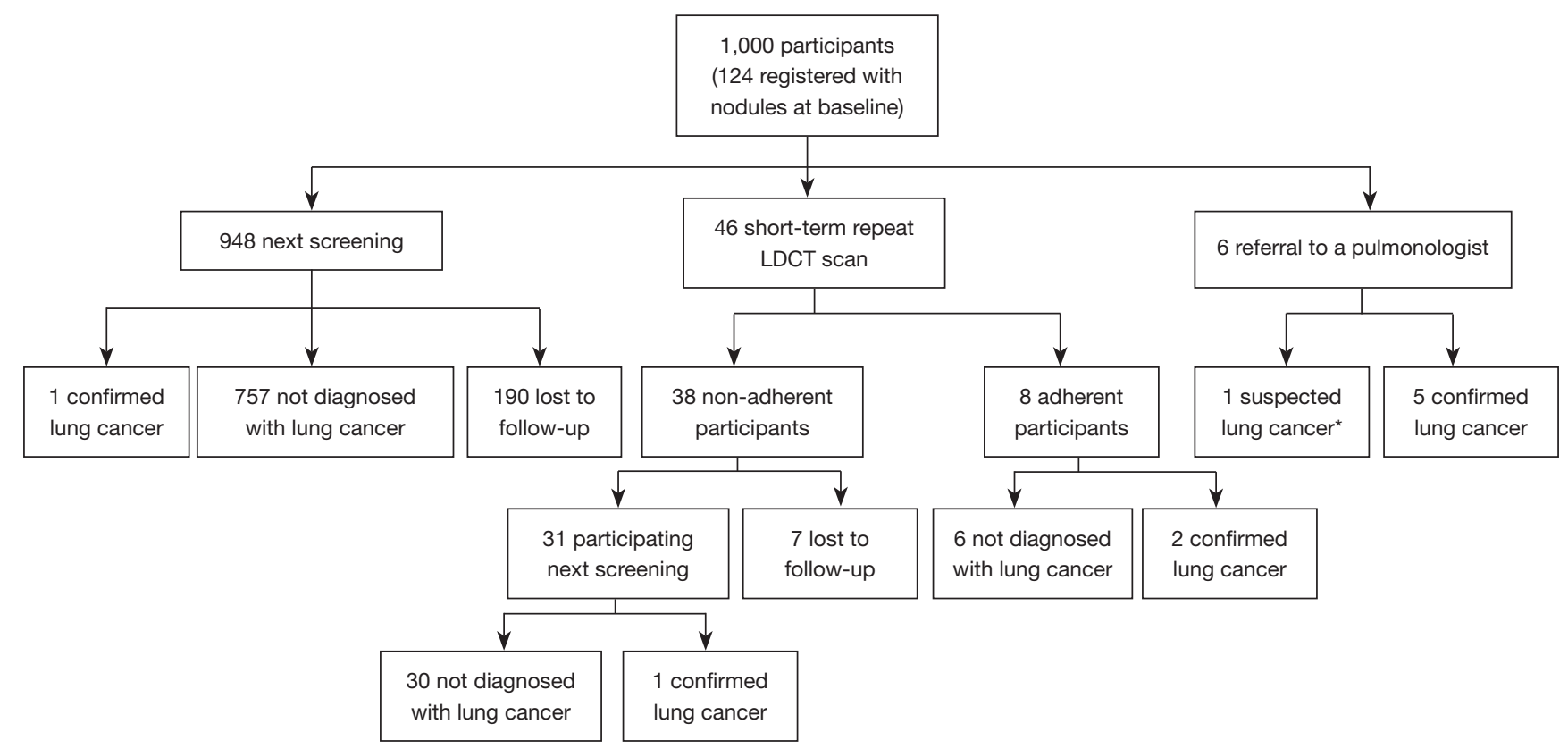

Figure 3 The actual management for all participants and lung cancer diagnosis until (including) the second round screening. *, suspected lung cancer according to the radiologist but without confirmation from histopathology. LDCT, low-dose computed tomography.

with lung cancer were detected through screening. One patient (No. 8) with a solid malignant nodule required short-term follow-up by NCCN protocol but required referral for further work-up by EUPS volume protocol, which means that EUPS volume protocol would detect this malignant lesion earlier (Table 2).

\section{Sensitivity analysis}

Compared to applying the average diameter rounded to the nearest whole number, applying the average diameter with one decimal fraction to the NCCN protocol led to fewer solid nodules (32, $22.2 \%$ vs. 49, 36.0\%) being classified as intermediate nodules (Table S2). Consequently, fewer participants would require short-term repeat CT scan (32, $3.2 \%$ vs. 45, 4.5\%; Table S3).

\section{Discussion}

In this study, we compared the management of participants when applying NCCN and EUPS protocols in a general Chinese population. We found that, in LDCT lung cancer screening setting, the number of referrals based on the EUPS volume protocol was 3 times higher than that based on the NCCN protocol; the number of short-term repeat scan based on the EUPS diameter protocol was double compared to that based on the EUPS volume protocol. One out of the 10 lung cancer patients would have been diagnosed earlier if followed the EUPS volume protocol.

There are some explanations for the larger number of referrals according to the EUPS volume protocol as compared to the NCCN protocol (1.9\% vs. $0.6 \%)$. The main explanation was that the EUPS protocol recommends a less stringent threshold in the definition of a positive nodule (volume $\geq 300 \mathrm{~mm}^{3}$ ) than the NCCN protocol (diameter $\geq 15 \mathrm{~mm}$ ). If assuming that nodules are spherical, the positive volume threshold is equivalent to $8.3 \mathrm{~mm}$ in diameter, which is much lower than the definition of the NCCN protocol. Therefore, more nodules required referral to a pulmonologist in the EUPS protocols in this study. In addition, the approach for measuring nodule diameter might contribute to the difference in number of referrals. The manual diameter measurements were smaller than the semi-automated diameter measurement in this study, suggesting underestimation of referral rate based on manual measurements.

Using semi-automated measurements of volume and diameter, at nodule level, the EUPS volume protocol was associated with a lower number of combined intermediate and positive nodules than the EUPS diameter protocol (at 
Table 2 Characteristics of participants diagnosed with lung cancer until (including) the second screening round

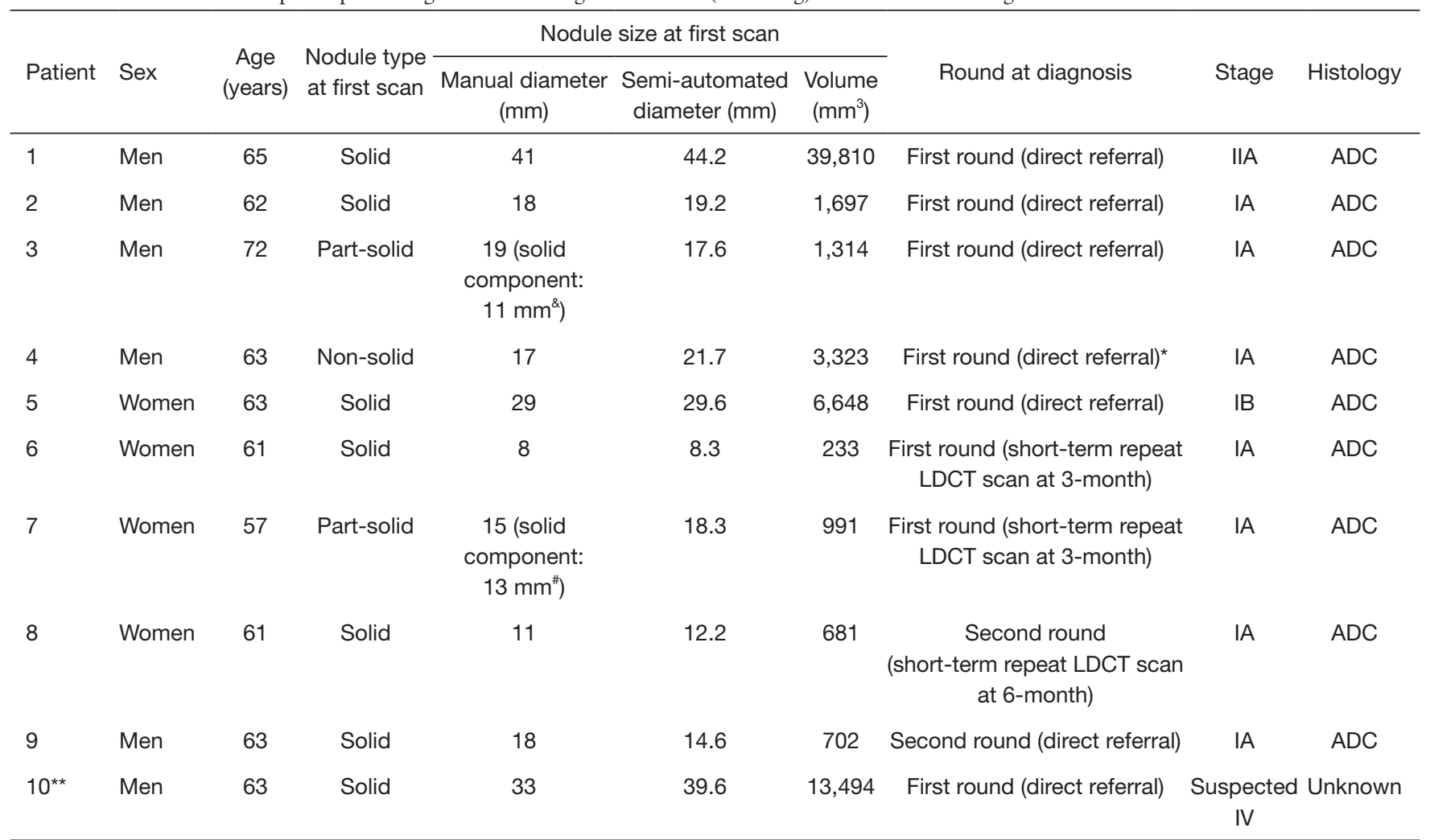

\&, solid component on mediastinal window was $8 \mathrm{~mm}$; ${ }^{*}$, although manual diameter $<19 \mathrm{~mm}$, referral was recommended based on other features of the nodule; ${ }^{*}$, solid component on mediastinal window was $6 \mathrm{~mm}$; ${ }^{*}$, suspected lung cancer according to the radiologist but without confirmation from histopathology. ADC, adenocarcinoma; LDCT, low-dose computed tomography.

participant level: $6.4 \%$ vs. $11.1 \%$, at nodule level: $42.9 \%$ vs. $84.5 \%$ ). This finding is consistent with another study, in which $34 \%$ of nodules were classified to Lung-RADS 3 or more when using semi-automated volume, and $52 \%$ were classified to these groups when using semi-automated diameter (10). Similar results were reported by NELSON study. At participant level, the proportion of short-term repeat LDCT scans and referrals using the EUPS volume thresholds was lower than using the equivalent EUPS diameter thresholds ( $8 \%$ vs. $22 \%$ for short-term repeat, $5 \%$ vs. $5 \%$ for referral) (11).

Applying the EUPS volume protocol, we found 4.5\% participants in need of a short-term repeat scan and $1.9 \%$ in need of referral to a pulmonologist, respectively. The figures in the first screening round of NELSON study showed $8 \%$ short-term repeat scans and 5\% referrals (11). The lower proportion of short-term repeat scan and referral in our study is probably due to the fact that we included a lower risk population (general population aged between
40 and 74 years, including a substantial proportion of neversmokers) compared to NELSON study in which heavy smokers were included.

Applying the NCCN protocol, $0.6 \%$ participants needed referral. This finding is similar to a study performed in Shanghai (12) but lower than a study in Sichuan, China (13). According to the report of lung cancer screening in a general population in Shanghai, $0.6 \%$ of included participants required referral to a pulmonologist (diameter $\geq 15 \mathrm{~mm}$ ) (12). The lung cancer screening study in Sichuan reported $1.6 \%$ of participants with nodule $>15 \mathrm{~mm}$ (13). The higher proportion in the study in Sichuan is probably due to that the high risk population was included. Furthermore, as shown in the sensitivity analysis, when the average diameter was not rounded to the nearest whole number, the number of solid nodules being classified as intermediate decreased by $35 \%$ from 49 to 32 , which suggested a potential decrease in the number of unnecessary follow-up LDCT scans. 
Table 3 Management of participants and lung cancer diagnosis after the first LDCT scan at baseline screening round according to NCCN and EUPS protocols

\begin{tabular}{|c|c|c|c|}
\hline Management & NCCN protocol & EUPS diameter protocol & EUPS volume protocol \\
\hline Next screening & $949(94.9 \%)$ & 889 (88.9\%) & $936(93.6 \%)$ \\
\hline Short-term repeat (3/6 months) & $45(4.5 \%)$ & $97(9.7 \%)$ & $45(4.5 \%)$ \\
\hline Referral to a pulmonologist & $6(0.6 \%)$ & $14(1.4 \%)$ & $19(1.9 \%)$ \\
\hline \multicolumn{4}{|l|}{ Participants with solid nodule $(n=103)$} \\
\hline Next screening & $57(55.3 \%)$ & $13(12.6 \%)$ & $45(43.7 \%)$ \\
\hline Short-term repeat (3/6 months) & $42(40.8 \%)$ & $76(73.8 \%)$ & $43(41.7 \%)$ \\
\hline Referral to a pulmonologist & $4(3.8 \%)$ & $14(13.6 \%)$ & $15(14.6 \%)$ \\
\hline \multicolumn{4}{|l|}{ Participants with part-solid nodule $(n=5)$} \\
\hline Referral to a pulmonologist & 2 & 0 & 4 \\
\hline \multicolumn{4}{|l|}{ Participants with non-solid nodule $(n=16)$} \\
\hline Next screening & 16 & 0 & 15 \\
\hline Short-term repeat (3/6 months) & 0 & 16 & 1 \\
\hline Referral to a pulmonologist & 0 & 0 & 0 \\
\hline \multicolumn{4}{|l|}{ Participants without nodules $(\mathrm{n}=876)$} \\
\hline Next screening & 876 & 876 & 876 \\
\hline No. of lung cancer diagnosis until (including) second round & 10 & 10 & 10 \\
\hline
\end{tabular}

LDCT, low-dose computed tomography; NCCN, National Comprehensive Cancer Network; EUPS, European Position Statement.

Table 4 Disagreement of NCCN and EUPS protocols for management of participants after the first LDCT scan at baseline screening ( $\mathrm{n}=1,000)$

\begin{tabular}{|c|c|c|c|c|c|c|}
\hline NCCN protocol & \multicolumn{3}{|c|}{ EUPS diameter protocol } & \multicolumn{3}{|c|}{ EUPS volume protocol } \\
\hline $\begin{array}{l}\text { Short-term repeat } \\
\text { (3/6 months) }\end{array}$ & 0 & 35 & 10 & 3 & 29 & 13 \\
\hline
\end{tabular}

Wilcoxon test for paired sample: $\mathrm{P}<0.001$ for NCCN vs. EUPS diameter, $\mathrm{P}<0.001$ for NCCN vs. EUPS volume. NCCN, National Comprehensive Cancer Network; EUPS, European Position Statement; LDCT, low-dose computed tomography.

The strength of this study was the well-defined general population for lung cancer screening and the prospective study design. Furthermore, manually-measured diameters were collected in addition to semi-automated diameter and volume measurements and all of them were applied in the management protocols for comparison. There were also some limitations in this study. First, the lower threshold in the EUPS volume protocol results in more referrals for 
further work-up, while also allows the possibilities of earlier cancer diagnosis (such as the patient No. 8). But because of the small number of participants with lung cancer, the sensitivity and specificity of the protocols were not calculated and a reliable conclusion regarding the performance of the evaluated protocols could not be drawn with the present study. In addition, about $20 \%$ of participants were lost to follow-up, their cancer status cannot be determined. Second, the EUPS protocol was developed for the early diagnosis of lung cancer in a high risk population. The included general population may not be eligible for such a protocol due to the low risk of lung cancer. Third, EUPS volume protocol was developed for solid nodule only. In this study, the volume thresholds for solid nodule were also applied for part-solid nodule. But even restricting the analysis in participants with solid nodule, the conclusion remained. Fourth, the main strength of EUPS volume-based protocol was the high sensitivity in detecting the increase of tumor growth, while we could not evaluate that in this Chinese population due to lack of the volume measurements from a second scan. It will be evaluated in our future studies. Fifth, the manual and semi-automatic measuring for nodule size were performed by different radiologists. The inter-reader variability was not evaluated in this study, but could affect the manual diameter and volume assessment of a lung nodule, especially, the inter-reader variability for semi-automated volume measurements is $11 \%$ lower than that for manual diameter measurements (14).

In conclusion, in a Chinese general population, the EUPS volume protocol leads to a higher pulmonologist referral rate than the NCCN protocol in LDCT lung cancer screening setting due to the less stringent criteria for referral. The less stringent criteria in the EUPS volume protocol are also associated with a probability of earlier cancer diagnosis. EUPS volume protocol is associated with a lower rate of short-term repeat LDCT scan and referral compared with the EUPS diameter protocol. In our future study, more data in lung cancer diagnosis will be available to allow assessing the performance of the evaluated protocols in a general population. Potentially, both diameter and volume protocols should be adjusted for low risk population, given that the referral rate may be too high in comparison to number of lung cancers found in a general population compared to a high risk population.

\section{Acknowledgments}

We acknowledge JM, DM, ZL and QD for the first reading of the LDCT images. YD is grateful for the $\mathrm{PhD}$ financial support from China Scholarship Council (CSC file No. 201708340072).

Funding: The study is a part of NELCIN-B3 project. The NELCIN-B3 project is funded by Ministry of The Science and Technology of the People's Republic of China (grant number: 2016YFE0103000) and The Royal Netherlands Academy of Arts and Sciences (grant number: PSA_SA_ BD_01). These funding sources had no role in the design, data collection, management, analysis, interpretation, preparation, review, approval of the manuscript, and decision to submit the manuscript for publication.

\section{Footnote}

Reporting Checklist: The authors have completed the STARD reporting checklist. Available at https://dx.doi. org/10.21037/jtd-21-1312

Data Sharing Statement: Available at https://dx.doi. org/10.21037/jtd-21-1312

Conflicts of Interest: All authors have completed the ICMJE uniform disclosure form (available at https://dx.doi. org/10.21037/jtd-21-1312). The authors have no conflicts of interest to declare.

Ethical Statement: The authors are accountable for all aspects of the work in ensuring that questions related to the accuracy or integrity of any part of the work are appropriately investigated and resolved. The study was conducted in accordance with the Declaration of Helsinki (as revised in 2013). The study was approved by Committee board on Ethics of Biomedicine Research of Second Military Medical University (No. 2018SL028) and informed consent was taken from all the participants.

Open Access Statement: This is an Open Access article distributed in accordance with the Creative Commons Attribution-NonCommercial-NoDerivs 4.0 International License (CC BY-NC-ND 4.0), which permits the noncommercial replication and distribution of the article with the strict proviso that no changes or edits are made and the 
original work is properly cited (including links to both the formal publication through the relevant DOI and the license). See: https://creativecommons.org/licenses/by-nc-nd/4.0/.

\section{References}

1. Sadate A, Occean BV, Beregi JP, et al. Systematic review and meta-analysis on the impact of lung cancer screening by low-dose computed tomography. Eur J Cancer 2020;134:107-14.

2. National Lung Screening Trial Research Team; Aberle DR, Adams AM, et al. Reduced lung-cancer mortality with low-dose computed tomographic screening. N Engl J Med 2011;365:395-409.

3. de Koning HJ, van der Aalst CM, de Jong PA, et al. Reduced Lung-Cancer Mortality with Volume CT Screening in a Randomized Trial. N Engl J Med 2020;382:503-13.

4. Bach PB, Mirkin JN, Oliver TK, et al. Benefits and harms of CT screening for lung cancer: a systematic review. JAMA 2012;307:2418-29.

5. American College of Radiology. Lung Rads. Available online: https://www.acr.org/Clinical-Resources/ Reporting-and-Data-Systems/Lung-Rads

6. National Comprehensive Cancer Network. Lung cancer screening. version 2.2018. 2017. Available online: https://www.nccn.org/guidelines/guidelinesdetail? category $=2 \& \mathrm{id}=1441$

7. Oudkerk M, Devaraj A, Vliegenthart R, et al. European position statement on lung cancer screening. Lancet Oncol 2017;18:e754-66.

8. Du Y, Zhao Y, Sidorenkov G, et al. Methods of computed tomography screening and management of lung cancer in Tianjin: design of a population-based cohort study. Cancer Biol Med 2019;16:181-8.

9. Du Y, Li Q, Sidorenkov G, et al. Computed Tomography Screening for Early Lung Cancer, COPD and Cardiovascular Disease in Shanghai: Rationale and Design of a Population-based Comparative Study. Acad Radiol 2021;28:36-45.

10. Gierada DS, Rydzak CE, Zei M, et al. Improved Interobserver Agreement on Lung-RADS Classification of Solid Nodules Using Semiautomated CT Volumetry. Radiology 2020;297:675-84.

11. Horeweg N, van Rosmalen J, Heuvelmans MA, et al. Lung cancer probability in patients with CT-detected pulmonary nodules: a prespecified analysis of data from the NELSON trial of low-dose CT screening. Lancet Oncol 2014;15:1332-41.

12. Fan L, Wang Y, Zhou Y, et al. Lung Cancer Screening with Low-Dose CT: Baseline Screening Results in Shanghai. Acad Radiol 2019;26:1283-91.

13. Liu Y, Luo H, Qing H, et al. Screening baseline characteristics of early lung cancer on low-dose computed tomography with computer-aided detection in a Chinese population. Cancer Epidemiol 2019;62:101567.

14. Han D, Heuvelmans MA, Oudkerk M. Volume versus diameter assessment of small pulmonary nodules in CT lung cancer screening. Transl Lung Cancer Res 2017;6:52-61.
Cite this article as: $\mathrm{Du} \mathrm{Y,} \mathrm{Li} \mathrm{Y,} \mathrm{Dorrius} \mathrm{MD,} \mathrm{Sidorenkov} \mathrm{G,}$ Vonder M, Vliegenthart R, Heuvelmans MA, Cui X, Ye Z, de Bock GH. Comparison of National Comprehensive Cancer Network and European Position Statement protocols for nodule management in low-dose computed tomography lung cancer screening in a general Chinese population. J Thorac Dis 2021;13(12):6855-6865. doi: 10.21037/jtd-21-1312 


\section{Supplementary}

Table S1 Classification of screen-detected lung nodules at the first LDCT scan of baseline screening using NCCN and EUPS protocols

\begin{tabular}{|c|c|c|c|c|c|c|}
\hline \multirow[b]{2}{*}{ Nodule classification } & \multicolumn{2}{|c|}{ NCCN protocol } & \multicolumn{2}{|c|}{ EUPS diameter protocol } & \multicolumn{2}{|c|}{ EUPS volume protocol } \\
\hline & $\begin{array}{l}\text { Diameter threshold } \\
\qquad(\mathrm{mm})\end{array}$ & $\begin{array}{c}\text { Manual } \\
\text { diameter*, } \\
\text { number (\%) }\end{array}$ & Threshold (mm) & $\begin{array}{c}\text { Semi-automated } \\
\text { diameter } \\
\text { number }(\%)\end{array}$ & Threshold $\left(\mathrm{mm}^{3}\right)$ & $\begin{array}{c}\text { Semi-automated } \\
\text { volume, } \\
\text { number (\%) }\end{array}$ \\
\hline \multicolumn{7}{|l|}{ Solid nodule } \\
\hline Negative nodule & $\leq 5$ & $91(63.2)$ & $<5$ & $25(17.4)$ & $<100$ & $78(54.2)$ \\
\hline Intermediate nodule & $\geq 6 \& \leq 14$ & $49(34.0)$ & $\geq 5 \&<10$ & $104(72.2)$ & $\geq 100 \&<300$ & $48(33.3)$ \\
\hline Positive nodule & $\geq 15$ & $4(2.8)$ & $\geq 10$ & $15(10.4)$ & $\geq 300$ & $18(12.5)$ \\
\hline \multicolumn{7}{|l|}{ Part-solid nodule } \\
\hline Negative nodule & $\leq 5$ & $0(0.0)$ & $<5$ & $0(0.0)$ & $<100$ & $0(0.0)$ \\
\hline Intermediate nodule & $\geq 6$ with solid part $\leq 7$ & $3(60.0)$ & $\geq 5$ & $5(100.0)$ & $\geq 100 \&<300$ & $1(20.0)$ \\
\hline Positive nodule & Solid part $\geq 8$ & $2(40.0)$ & - & - & $\geq 300$ & $4(80.0)$ \\
\hline \multicolumn{7}{|l|}{ Non-solid nodule } \\
\hline Negative nodule & $\leq 19$ & $19(100.0)$ & $<5$ & $1(5.3)$ & $<1,125$ & $18(94.7)$ \\
\hline Intermediate nodule & $\geq 20$ & $0(0.0)$ & $\geq 5$ & $18(94.7)$ & $\geq 1,125$ & $1(5.3)$ \\
\hline \multicolumn{7}{|l|}{ Overall } \\
\hline Negative nodule & - & $110(65.5)$ & - & $26(15.5)$ & - & $96(57.1)$ \\
\hline Intermediate nodule & - & $52(30.9)$ & - & $127(75.6)$ & - & $50(29.8)$ \\
\hline Positive nodule & - & $6(3.6)$ & - & $15(8.9)$ & - & $22(13.1)$ \\
\hline
\end{tabular}

${ }^{*}$, average diameter rounded to the nearest whole number; ${ }^{\$}$, average diameter. LDCT, low-dose computed tomography; NCCN, National Comprehensive Cancer Network; EUPS, European Position Statement.

Table S2 Classification of screen-detected lung nodules at the first LDCT scan of baseline screening using NCCN protocol (without rounding the average diameter to the nearest whole number)

\begin{tabular}{lcc}
\hline Nodule classification & Diameter threshold $(\mathrm{mm})$ & Manual diameter ${ }^{\star}$, number $(\%)$ \\
\hline Solid nodule & $<6$ & $108(75.0)$ \\
Negative nodule & $\geq 6 \&<15$ & $32(22.2)$ \\
Intermediate nodule & $\geq 15$ & $4(2.8)$ \\
Positive nodule & $<6$ & $0(0.0)$ \\
Part-solid nodule & $\geq 6$ with solid part $<8$ & $3(60.0)$ \\
Negative nodule & Solid part $\geq 8$ & $2(40.0)$ \\
Intermediate nodule & & \\
Positive nodule & $<20$ & $19(100.0)$ \\
Non-solid nodule & $\geq 20$ & $0(0.0)$ \\
Negative nodule & & \\
Intermediate nodule & - & $127(75.6)$ \\
Overall & - & $35(20.8)$ \\
Negative nodule & - & $6(3.6)$ \\
Intermediate nodule & & \\
Positive nodule & &
\end{tabular}

*, average diameter not rounded to the nearest whole number. LDCT, low-dose computed tomography; NCCN, National Comprehensive Cancer Network. 
Table S3 Management of participants and lung cancer diagnosis after the first LDCT scan at baseline screening round according to NCCN protocol (without rounding the average diameter to the nearest whole number)

\begin{tabular}{lc}
\hline Management & NCCN protocol \\
\hline All participants ( $\mathrm{n}=1,000)$ & $962(96.2 \%)$ \\
Next screening & $32(3.2 \%)$ \\
Short-term repeat (3/6 months) & $6(0.6 \%)$ \\
Referral to a pulmonologist & \\
Participants with solid nodule ( $\mathrm{n}=103)$ & $70(68.0 \%)$ \\
Next screening & $29(28.2 \%)$ \\
Short-term repeat (3/6 months) & $4(3.8 \%)$ \\
Referral to a pulmonologist & 0 \\
Participants with part-solid nodule ( $\mathrm{n}=5)$ & 3 \\
Next screening & 2 \\
Short-term repeat (3/6 months) & \\
Referral to a pulmonologist & 16 \\
Participants with non-solid nodule ( $\mathrm{n}=16)$ & 0 \\
Next screening & 0 \\
Short-term repeat (3/6 months) & \\
Referral to a pulmonologist & \\
Participants without nodules $(\mathrm{n}=876)$ & \\
Next screening & \\
No. of lung cancer diagnosis until (including) & \\
second round & \\
\hline LCT low-dose computed tomography; NCCN, National
\end{tabular}

LDCT, low-dose computed tomography; NCCN, National Comprehensive Cancer Network. 